\title{
Metabolic mechanism of Saccharomyces cerevisiae under different physiological conditions based on non-stationary $13 \mathrm{C}$ metabolic flux analysis
}

Huan Li (D 1270504921@qq.com)

East China University of Science and Technology https://orcid.org/0000-0001-7581-1666

Min Chen

East China University of Science and Technology

Peng Liu

East China University of Science and Technology

Shuai Wang

East China University of Science and Technology

JY Xia

East China University of Science and Technology

Research

Keywords: Saccharomyces cerevisiae, INST-13C-MFA, Crabtree effect, central carbon metabolic, metabolic flux

Posted Date: May 28th, 2020

DOl: https://doi.org/10.21203/rs.3.rs-26832/v1

License: (c) (i) This work is licensed under a Creative Commons Attribution 4.0 International License.

Read Full License 
Title page:

Metabolic mechanism of Saccharomyces cerevisiae under different physiological conditions based on non-stationary ${ }_{13} \mathrm{C}$ metabolic flux analysis

Huan L : East China university of science and technology

Min C : East China university of science and technology

Peng L : East China university of science and technology

Shuai W : East China university of science and technology

JY Xia* : East China university of science and technology

(*: the corresponding author)

The email address of the corresponding author: jyxia@ecust.edu.cn 
Abstract: Crabtree effect is well known for Saccharomyces cerevisiae, and is defined as glucose-induced repression of respiratory flux. Even though a number of hypotheses have been formulated, its triggering mechanisms are still unknown. At present, the information about intracellular metabolic flux can be obtained by the ${ }_{13} \mathrm{C}$ isotope labeling experiments. $13 \mathrm{C}$ metabolic flux analysis(13C-MFA) is a traditional method for calculating metabolic flux based on isotopic steady state. Another new method (INST-13C-MFA: Isotopically nonstationary metabolic flux analysis) based on isotope non-steady state is being used by researchers. In this review, we have chemostatized $S$. cerevisiae at three different dilution rates $(\mathrm{D}=0.12,0.22,0.32 \mathrm{~h}-1)$ and obtained the metabolic flux distribution of the intracellular central carbon metabolic of S. cerevisiae using INST-13C-MFA. Combined with the metabolome and metabolic fluxome data, we found obvious metabolic flux shift under the three different physiological states. In this process, pyruvate decarboxylase, ethanol dehydrogenase and acetyl-CoA synthase(AcCoA) catalyzed reactions were key points. Negative correlation between relative flux of embden meyerh of pathway(EMP) and tricarboxylic acid cycle(TCA) and biomass yield, while positive correlation for pentose phosphate pathway(PPP) were observed. Yield of acetate and glycerol did not change significantly, while that of ethanol increased sharply. In the central carbon metabolism (CCM), most of the carbon flux (70\%) was 
directed to the EMP. At the same time, the energy charge increased with dilution rate, and the cell's energy supply mode gradually shifted from oxidative respiration to substrate level phosphorylation mode.

Key words: Saccharomyces cerevisiae; INST-13C-MFA; Crabtree effect; central carbon metabolic; metabolic flux;

\section{Introduction}

The constantly decreasing amount of fossil resources, global warming as well as increasing public demand for sustainable production processes drive the research in the field of bio-based alternatives (Burk and Van Dien 2016). The use of microorganisms for different kinds of applications has a long tradition, starting with alcoholic fermentation as well as acetic acid production (Zhang, Sun, and Ma 2017; Zheng et al. 2018). The product scope has broadened tremendously over the past decades to various high-volume chemicals, materials, and fuels, and high-value molecules for human health and nutrition (Schwechheimer et al. 2016; Becker and Wittmann 2012; Becker et al. 2015; Becker and Wittmann 2016). For Saccharomyces cerevisiae, even under aerobic conditions, it is an industrial microorganism that still undergoes ethanol fermentation when the glucose concentration is high (De Deken 1966). Simultaneously, the yeast Saccharomyces cerevisiae is one of the major workhorses used in biotechnology for producing pharmaceuticals, 
biofuels (Hashem and Darwish 2010), and bulk chemicals (Willke and Vorlop 2004). More importantly, S. cerevisiae is also an important model organism for studying the physiology (Pereira, Eleutherio, and Panek 2001), genetics and metabolic mechanisms of eukaryotes (Castrillo et al. 2007). As we all know, metabolism is among the most strongly conserved processes across all domains of life and is crucial for both bioengineering and disease research (Hackett et al. 2016).Estimating rates of reactions of metabolic network accurately is crucial for metabolic application (Suarez-Mendez et al. 2016). This is because that the knowledge of intracellular reaction rates (fluxes) is crucial to understand how cells utilize nutrients and how they adapt the fluxes in response to environmental or genetic perturbations (Lehnen, Ebert, and Blank 2017). Crabtree effect is well known for Saccharomyces cerevisiae, and is defined as glucose-induced repression of respiratory flux. Even though a number of hypotheses have been formulated, its triggering mechanisms are still unknown. So, Crabtree effect has caused widespread concern, and it is significance to revealing its mechanism. The investigation of intracellular reaction rates has become accessible to a wide range of scientists, rather than exclusively experts, and has been applied to expand detailed knowledge on cellular physiology (Blank, Lehmbeck, and Sauer 2005; Long and Antoniewicz 2014; Petersen et al. 2000), as well to guide metabolic engineering (Bartek et al. 2011; Stephanopoulos 1999; Toya 
and Shimizu 2013) and biomedical research (Boghigian et al. 2010).

At present, the most efficient and accurate means of obtaining the data of intracellular metabolic flux is the ${ }_{13} \mathrm{C}$ isotope labeling experiments. 13C-metabolic flux analysis (13C-MFA) has emerged over the past 20 years as an important approach to assess in vivo metabolic phenotypes (Sauer 2006; Wiechert 2001). In 13C-MFA, after feeding of a ${ }_{13} \mathrm{C}$-labeled carbon source into a cell culture, amino acid or intermediates are extracted and subjected to mass spectrometric analysis. It relies on least-squares regression of isotope labeling measurements and extracellular exchange rates to reconstruct comprehensive flux maps that depict the flow of carbon throughout intracellular metabolism (Young 2014). Based on the labeled information obtained by the isotope labeling experiment (wet experiment), the optimal evaluation value of the metabolic flux is obtained by computer iterative calculation and continuous fitting. Therefore, we need some software platforms for calculating the metabolic flux. At present, in order to promote the wider application of ${ }_{13} \mathrm{C}-\mathrm{MFA}$ in the construction of metabolic network models and the calculation of metabolic fluxes, several publicly available software packages have been developed, which have: 13CFLUX2 (Weitzel et al. 2013), Metran (Yoo et al. 2008), OpenFLUX (Quek et al. 2009), INCA (Young 2014), OpenMebius (Kajihata et al. 2014).

In our review, OpenMebius (Open source software for Metabolic 
flux analysis) software platform was used to calculate the intracellular metabolic flux of Saccharomyces cerevisiae at different dilution rates. When the metabolic steady state is reached, OpenMebius has been developed to perform INST-13C-MFA(isotopically nonstationary ${ }_{13} \mathrm{C}$ metabolic flux analysis) and conventional ${ }_{13} \mathrm{C}-\mathrm{MFA}$ (isotopically stationary ${ }_{13} \mathrm{C}$ metabolic flux analysis) using a user-defined metabolic model (Kajihata et al. 2014). In OpenMebius, A metabolic model $M$ can be automatically generated from a metabolic pathway and carbon transition network. Under the metabolic network model $M$, combined with the labeling information of the ${ }_{13} \mathrm{C}$ isotope, the optimal metabolic flux distribution map was obtained by nonlinear fitting. In our experiments, INST-13C-MFA (see Fig 1) was used to calculate the metabolic flux of $S$. cerevisiae at three different dilution rates $(\mathrm{D}=0.12$, $0.22,0.32 \mathrm{~h}-1)$. Combining the data of metabolites and metabolic flux, we try to explain the mechanism of Crabtree effect and found that $S$. cerevisiae undergoes metabolic migration of intracellular central carbon metabolic under three different physiological conditions. Moreover, the metabolic flux of the EMP pathway, the PP pathway, and the TCA cycle (when the glucose uptake rate is set to 1) has a certain relationship with the yield of the biomass. 


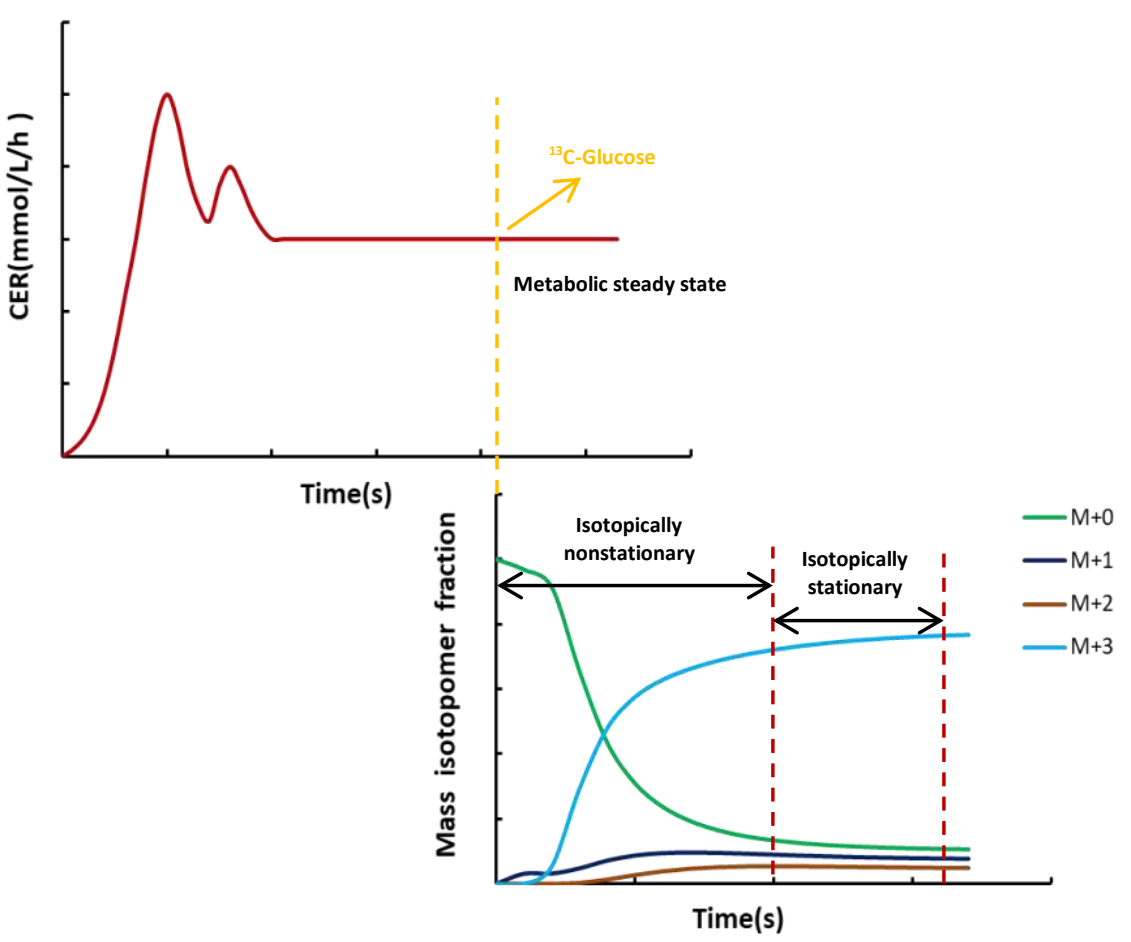

Fig1: Metabolic steady state and isotopically stationary. The isotopic labeling experiment is performed under metabolic steady state (the yellow dotted line indicates that metabolic steady is achieved after 5 residence times). After feeding $13 \mathrm{C}$-labelde glucose, isotopic labeling enrichment changes in a time-dependent manner and then reaches a stationary condition. Whereas $S$. cerevisiae are sampled under isotopically stationary conditions in conventional ${ }_{13} \mathrm{C}-\mathrm{MFA}$, time courses of isotopic labeling enrichment during an isotopically transient state are used for INST-13C-MFA.

\section{Material and methods}

\subsection{Strain and culture conditions}

We used the Saccharomyces cerevisiae CEN PK 113-7D strain purchased from EUROSCAR, activated in YPD solid medium, and the activated strain was stored in $-80^{\circ} \mathrm{C}$ glycerol tubes. A bioreactor (Model: 
Multifors Bacteria) manufactured by INFORS was used to culture the yeast. Trace metal solution in Delft medium: $3 \mathrm{~g} / \mathrm{L} \mathrm{FeSO}_{4} \cdot 7 \mathrm{H}_{2} \mathrm{O}, 4.5 \mathrm{~g} / \mathrm{L}$ $\mathrm{ZnSO}_{4} \bullet 7 \mathrm{H}_{2} \mathrm{O}, \quad 4.5 \mathrm{~g} / \mathrm{L} \quad \mathrm{CaCl}_{2} \bullet 2 \mathrm{H}_{2} \mathrm{O}, \quad 1 \mathrm{~g} / \mathrm{L} \quad \mathrm{MnCl}_{2} \bullet 4 \mathrm{H}_{2} \mathrm{O}, \quad 0.3 \mathrm{~g} / \mathrm{L}$ $\mathrm{CoCl}_{2} \bullet 6 \mathrm{H}_{2} \mathrm{O}, 0.3 \mathrm{~g} / \mathrm{LCuSO}_{4} \cdot 5 \mathrm{H}_{2} \mathrm{O}, 0.4 \mathrm{~g} / \mathrm{L} \mathrm{NaMoO}_{4} \cdot 2 \mathrm{H}_{2} \mathrm{O}, 1 \mathrm{~g} / \mathrm{L} \mathrm{H}_{3} \mathrm{BO}_{3}$, $0.1 \mathrm{~g} / \mathrm{L} \mathrm{KI}, 19 \mathrm{~g} / \mathrm{L}$ Na2EDTA $2 \mathrm{H}_{2} \mathrm{O}$; Vitamin solution in Delft medium: $0.05 \mathrm{~g} / \mathrm{L}$ d-Biotin, $1 \mathrm{~g} / \mathrm{L}$ D-Pantothenic acid hemicalcium salt, $1 \mathrm{~g} / \mathrm{L}$ Thiamin-HCl, 1 g/L Pyridoxin-HCl, 1 g/L Nicotinic acid, 0.2 g/L 4-aminobenzoic acid, $25 \mathrm{~g} / \mathrm{L}$ m-Inositol. The solution of Saccharomyces cerevisiae was taken from the glycerol tube and streaked on the plate of the YPD solid medium, placed in a $30^{\circ} \mathrm{C}$ incubator, and cultured for 48 hours; Pick a single colony of yeast from the plate and inoculate it in a tube containing $3 \mathrm{ml}$ of Delft medium [7.5 g/L $\left(\mathrm{NH}_{4}\right)_{2} \mathrm{SO}_{4}, 14.4 \mathrm{~g} / \mathrm{L}$ $\mathrm{KH}_{2} \mathrm{PO}_{4}, 0.5 \mathrm{~g} / \mathrm{L} \mathrm{MgSO}_{4} \bullet 7 \mathrm{H}_{2} \mathrm{O}, 10 \mathrm{~g} / \mathrm{L}$ glucose, $1 \mathrm{ml} / \mathrm{L}$ vitamin solution, $2 \mathrm{ml} / \mathrm{L}$ trace metal solution], the tube was incubated for 12 hours in a shaker at a speed of $220 \mathrm{rpm}$ and a temperature of $30^{\circ} \mathrm{C}$. After that, transfer the fermentation broth in the test tube to a shake flask containing $100 \mathrm{ml}$ of Delft medium that is consistent with the medium in the test tube, the shake flask was incubated for 12 hours in a shaker at a speed of $220 \mathrm{rpm}$ and a temperature of $30^{\circ} \mathrm{C}$. Finally, the fermentation broth in the shake flask is transferred to a bioreactor containing $600 \mathrm{ml}$ of Delft medium [5 g/L (NH4)2SO 3 , 3 g/L KH${ }_{2} \mathrm{PO}_{4}, 0.5 \mathrm{~g} / \mathrm{L} \mathrm{MgSO}_{4} \cdot 7 \mathrm{H}_{2} \mathrm{O}, 10 \mathrm{~g} / \mathrm{L}$ glucose, $1 \mathrm{ml} / \mathrm{L}$ vitamin solution, $1 \mathrm{ml} / \mathrm{L}$ trace metal solution]. The 
aeration was performed using pressurized air at $0.6 \mathrm{~L} / \mathrm{min}$ (approx. 1vvm) and a stirrer speed of $700 \mathrm{rpm}$. The broth was maintained at $\mathrm{PH} 5.0$ by adding $2 \mathrm{M} \mathrm{KOH}$, the temperature was controlled at $30^{\circ} \mathrm{C}$ and pressure was kept at 0.3 bar.

\subsection{Chemostat cultivation}

When the dissolved oxygen rises and the carbon-dioxide escape rate(CER) falls for the second time (the two occur at the same time), that indicates reactions reach the end of the batch fermentation stage due to the "secondary growth" phenomenon of Saccharomyces cerevisiae. After that it begins to transfer to the chemostat culture. The composition of Delft medium added into bioreactor is the same as that of the batch fermentation medium during the chemostat culture, besides the concentration of glucose is changed to $7.5 \mathrm{~g} / \mathrm{L}$. The rest of the fermentation parameters are consistent with the batch fermentation. Ethanol was not added to the medium since no oscillations were observed under these conditions. We performed chemostatization experiments at three different dilution rates $(\mathrm{D}=0.12,0.22,0.32 \mathrm{~h}-1)$. After culturing five elution volumes, we considered that metabolic steady was reached (van Eunen et al. 2011). At this time, the parameters such as CER, oxygen escape rate (OUR), and dissolved oxygen are basically unchanged, these phenomena also prove the state of achieving metabolic homeostasis. At the same time, we began to switch the substrates to a medium containing 
${ }_{13} \mathrm{C}$-labeled glucose. Ingredients in the medium are consistent with the medium of chemostat, the only difference between these two media was that glucose labeled with ${ }_{13} \mathrm{C}$ and $\mathrm{U}-{ }_{13} \mathrm{C}: 1-13 \mathrm{C}=4: 1$. The feed rate and parameters of fermentation remain constant, ensuring that the Saccharomyces cerevisiae in the bioreactor is still stay the state of metabolic steady after switching substrates. ${ }_{13} \mathrm{C}$-glucose is maintained for $1 / 10$ elution volumes, we sampling from the broth rapidly to obtain data about the changes of isotopically labeled information over time during this process.

\subsection{Rapid sampling}

An in-house-made rapid sampling device was applied for the rapid sampling after switching the substrate. The Single Chip Microcomputer technique was employed to control the opening and closure of three valves. The device was optimized so that it takes less than $1 \mathrm{~s}$ to sample a volume of $\sim 1 \mathrm{~mL}$ broth.

Considering that the turnover time of intracellular metabolites is very short(Sauer 2006), in order to accurately obtain the data of changes in the mass distribution vector (MDV) of intracellular metabolites over time, during the period of supplementation of ${ }_{13} \mathrm{C}$-glucose, we conducted sampling densely in the early stage: first 40 s, sampling every 5 s. Taking samples every 20 s and takes about 5 samples in the middle. The interval between the later sampling is longer: sampling every 1 minute, or 
sampling every 5 minutes. The entire process of rapid sampling takes approximately 20 samples. At different dilution rates, the time of the rapid sampling process is also different. At the dilution rates $\mathrm{D}=0.12,0.22$, $0.32 \mathrm{~h}-1$, the rapid sampling process lasts for 60,30 , and 20 minutes, respectively.

\subsection{Data acquisition}

The concentration of biomass was determined by a gravimetrical method (dry weight). When metabolic steady state is reached, $10 \mathrm{ml}$ of broth were filtered through a pre-dried and pre-weighted membrane $(0.8$ $\mu \mathrm{m}, 0.47 \mathrm{~mm})$. The filter containing the biomass on the surface was placed in a microwave oven whose power is $700 \mathrm{~W}$ and dried again for 3 min. After cooling to room temperature, it was weighed. We have done pre-experiment using this method before, and the weight of the filter is basically unchanged after $2.5 \mathrm{~min}$, it can be considered completely dry. The biomass concentration $\left(C_{x}\right)$ can be used to calculate the glucose consumption rate $\left(\mathrm{q}_{\mathrm{s}}\right)$ and the product rate of extracellular metabolites (qeth, qgly, qace) later.

\subsubsection{Extracellular metabolites}

In our experiments, the concentration of extracellular by-products was quantized, which determines the rate of exchange reactions and provides indispensable data for calculating metabolic fluxes. When reach the metabolic steady state, we believe that the concentrations of both 
intracellular and extracellular metabolites are in a stable state. For quantification of extracellular metabolite concentration, about $1.5 \mathrm{ml}$ of the broth was taken out from the bioreactor, placed in a pre-cooled $\left(-20^{\circ}\right.$ C) syringe, filtered through a $0.22 \mu \mathrm{m}$ filter (Mashego et al. 2006), and the filtrate was used for analysis of extracellular by-products and residual glucose. Extracellular (filtrate) glucose, ethanol, acetate and glycerol concentrations were determined by one/all of the following two methods: HPLC, or enzymatic assays (Canelas et al. 2011).

The extracellular glucose concentration was measured by a glucose reagent kit (Huili biotech co., LTD, China). The glucoamylase concentration was determined by NPG method. $11.5 \mu \mathrm{L}$ sample was mixed with $131.5 \mu \mathrm{L}$-NPG substrate (pre-warmed for 5 min at $37^{\circ} \mathrm{C}$, 2 g/L 4-Nitrophenyl- $\alpha$-d-glucopyranoside, Sigma N-1377). After incubation at $37^{\circ} \mathrm{C}$ for $20 \mathrm{~min}$, the reaction was stopped by adding $57 \mu \mathrm{L}$ $0.3 \mathrm{M} \mathrm{Na}_{2} \mathrm{CO}_{3}$ with immediate absorbance reading at $405 \mathrm{~nm}$ on a plate reader (MKIII plate reader, Thermo-Fisher, USA).

In this study, the extracellular metabolites detected are mainly ethanol, acetate and glycerol. The concentrations of extracellular metabolites were measured using HPLC, using an Agilent Technologies Hi-Plex H column $(300 \times 7.7 \mathrm{~mm})$ equipped with a guard column $(50 \times 7.7 \mathrm{~mm})$. The mobile phase used was $3 \mathrm{M} \mathrm{H}_{2} \mathrm{SO}_{4}$, with a flow rate of $0.4 \mathrm{~L} / \mathrm{min}$. 


\subsubsection{Intracellular metabolites}

For quantification of intracellular metabolites concentrations, about $1 \mathrm{ml}$ broth samples were rapidly withdrawn and quenched in pre-weighted tubes containing $20 \mathrm{ml}$ cold $\left(-40^{\circ} \mathrm{C}\right)$ pure methanol (Canelas et al. 2009) followed immediately by vigorous vortexing. The quenched samples were rapidly weighed and poured into a filtration device containing a cellulose membrane $(0.8 \mu \mathrm{m}, \quad 0.47 \mathrm{~mm})$. Subsequently, a vacuum was applied followed by an immediate additional washing step with $10 \mathrm{ml}$ cold methanol $\left(-40^{\circ} \mathrm{C}\right)$. The filter containing the cold washed biomass was then transferred into a 50 ml-centrifuge tube containing $20 \mathrm{ml}$ of preheated $\left(75^{\circ} \mathrm{C}\right)$ aqueous ethanol solution $(75 \%$ $\mathrm{v} / \mathrm{v}) .100 \mu \mathrm{L}$ of ${ }_{13} \mathrm{C}$ cell extract was added to the tube as an internal standard ( $\mathrm{Wu}$ et al. 2005). The tube containing the sample was then tightly closed, shaken vigorously, and placed into a water bath at $95^{\circ} \mathrm{C}$ during $3 \mathrm{~min}$ for intracellular metabolite extraction. After, the tubes were then cooled using ice bath and the filter was removed. This extract was then concentrated by complete evaporation of ethanol-water mixture under vacuum (Mashego et al. 2004). Finally, $600 \mu \mathrm{l}$ of milliQ water was added to the centrifuge tube, and after thoroughly mixing and dissolved, the solution was transferred to a $1.5 \mathrm{ml}$ EP tube placed into a screw-capped polypropylene vial and stored at $-80^{\circ} \mathrm{C}$ until further analysis. Samples were analyzed by LC -MS/MS (van Dam, Ras, and ten 
Pierick 2011; Sollner et al. 2009) and/or GC-MS (de Jonge et al. 2011;

Cipollina et al. 2009).

\subsubsection{Calculation methods}

The biomass specific uptake and secretion rates were reconciled using the approach of Verheijen (2010). A constant elemental biomass composition and molar weight of 26.4 gDCw-C-mol-1 was used for all conditions.

The biomass specific uptake and secretion rates (q: $\mathrm{mmol} / \mathrm{gDCw} / \mathrm{h})$, were calculated from the respective dynamic mass balance using polynomial fits for the measured biomass and extracellular metabolite concentrations as a function of time ( $\mathrm{i}=$ biomass, ethanol, acetate and glycerol):

$$
\frac{d C_{i}(t) V_{L}}{d t}=F_{L, \text { in }} C_{i, \text { in }}-F_{L, \text { out }} C_{i}(t)+q_{i}(t) C_{x}(t) V_{L}
$$

Here, $C_{i}$ is concentration of compound $\mathrm{i}$ in the reactor, $V_{L}$ is broth volume, $F_{L, \text { in }}$ is medium feed flow, $F_{L, \text { out }}$ is effluent flow, $q_{i}$ is biomass specific consumption/secretion rates for compound i, $C_{x}$ is biomass concentration.

The biomass specific $\mathrm{O}_{2}$ uptake and $\mathrm{CO}_{2}$ evolution rates were calculated from the pseudo steady state gas phase balances $\left(\mathrm{j}=\mathrm{O}_{2}, \mathrm{CO}_{2}\right)$ (where $\mathrm{F}_{g}$,out was obtained from the $\mathrm{F}_{\text {g,in }}$ and the $\mathrm{N}_{2}$ balance):

$$
0=F_{g, \text { in }} y_{j, \text { in }}-F_{g, \text { out }} y_{j, \text { out }}+q_{j}(t) C_{x}(t) V_{L}
$$

Where $C_{x}$ is biomass concentration, $V_{L}$ is broth volume, $F_{g, i n}$ is gas 
flow in, $F_{g, \text { out }}$ is gas flow out, $y_{j, \text { in }}$ is concentration of compound $\mathrm{j}$ in gas flow in, $y_{j, \text { out }}$ is concentration of compound $\mathrm{j}$ in gas flow out, $q_{j}$ is specific consumption/secretion rates for compound $\mathrm{j}$.

\subsubsection{Mass isotopomer measurements}

Samples for quantification of mass isotopomer enrichments were taken following the same procedure described above for concentration of extra- and intracellular metabolites with the exception that no ${ }_{13} \mathrm{C}$ cell extract was added. The mass isotopomer samples were then analyzed by LC -MS/MS (van Winden et al. 2005).

\subsubsection{Estimate metabolic flux}

Under the Linux operating system, OpenMebius is run using MATLAB (MathWork, Natick, MA, USA), which builds a metabolic model(see Supplementary material 1) based on metabolic network model and carbon atom migration model. Under this metabolic model, we need to provide data about the concentration of metabolites (all metabolites contained in the model) and the rate of exchange reactions. At different dilution rates, the rate of intermediates converted into biomass/amino acids in the exchange reaction is calculated based on the equation of bacterial synthesis. In this way, we can obtain the simulated value of a series of the mass distribution vector $\left(\mathrm{MDV}_{\mathrm{sim}}\right)$ of intracellular metabolites (Antoniewicz, Kelleher, and Stephanopoulos 2007; Kajihata et al. 2014). Finally, the MDV sim is compared with the experimental value 
$\mathrm{MDV}_{\exp }$ obtained by the labeling experiment to find the group which shows the optimal fitting effect, and that is the data of the metabolic flux we need. We made a simple summary explained the entire process of calculating metabolic flux, the steps of the method are shown in below (see Fig 2).

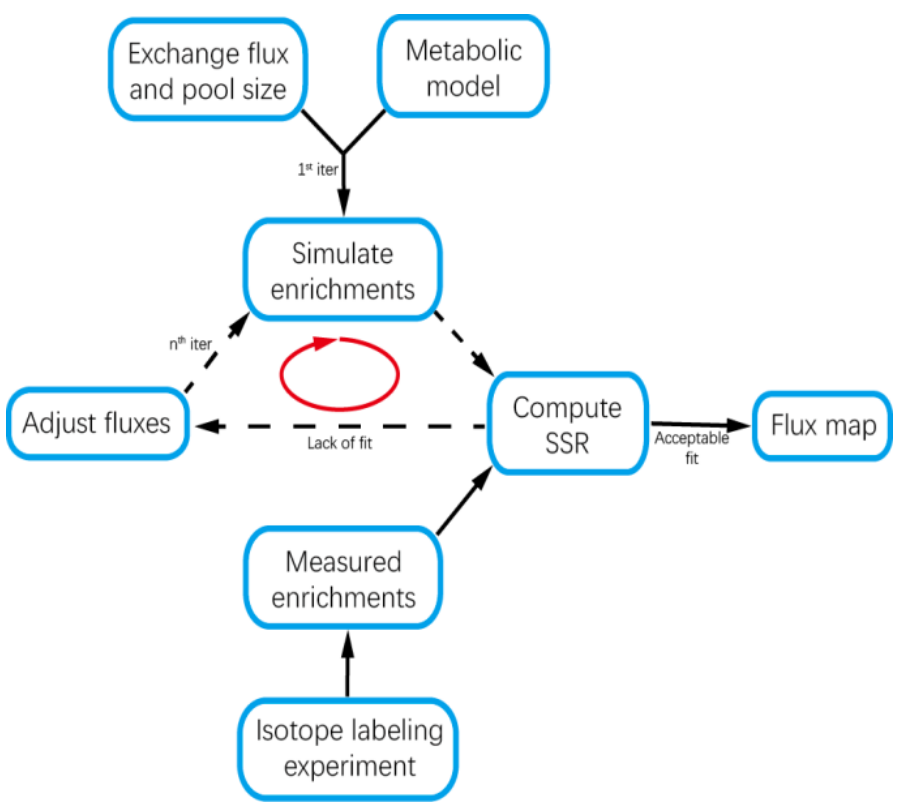

Fig 2: Schematic diagram of the calculation of metabolic flux by the INST-MFA method. Currently available software tools, namely OpenMebius, are used for model specification, flux estimation, and statistical analysis. Initial guesses of fluxes and pool sizes are needed for the first iteration (iter) only. The flux map generated is a snapshot of metabolism during the course of the isotope labeling experiments (ILEs).

\section{Results}

\subsection{Carbon-balance check}

At three different dilution rates, we performed extracellular sampling and measured the concentration of extracellular metabolites when 
metabolic steady state was reached. The data are shown in Table 1. It can be seen that a small portion of acetate and glycerol are secreted from the yeast at different dilution rates, and the concentration of them does not change significantly with changes in specific growth rate. For this, we calculated the $\mathrm{C}$-balance at these three dilution rates. When $\mathrm{D}=0.12,0.22$, $0.32 \mathrm{~h}-1$, the C-balance is $104.23 \%, 88.16 \%$, and $108.09 \%$, respectively. Among them, the C-balance at a dilution rate of $0.22 \mathrm{~h}-1$ is not strictly observed

Table 2 shows the specific formation/consumption rates (unit: $\mu$ mol gDCW-1 h-1) for each metabolites at different dilution rates. It can be seen that the specific consumption rate of glucose increases with the increase of specific growth rate. When D increases from $0.12 \mathrm{~h}-1$ to $0.32 \mathrm{~h}-1$, the specific consumption rate of glucose increases by nearly 3 times.

\section{Table 1}

Macroscopic measurements for different dilution rate.

\begin{tabular}{cccc}
\hline Dilution rate (h-1) & 0.12 & 0.22 & 0.32 \\
\hline Biomass concentration (gDCwL-1) & $3.42 \pm 0.01$ & $3.66 \pm 0.01$ & $2.44 \pm 0.01$ \\
Residual Glucose concentration (mM) & $0.36 \pm 0.03$ & $0.38 \pm 0.03$ & $1.37 \pm 0.05$ \\
Ethanol concentration (mM) & b.d. & b.d. & \\
Acetate concentration (mM) & $2.72 \pm 0.15$ & $1.35 \pm 0.09$ & $3.12 \pm 0.15$ \\
Glycerol concentration $(\mathrm{mM})$ & $1.35 \pm 0.17$ & $0.55 \pm 0.05$ & $1.24 \pm 0.10$ \\
\hline
\end{tabular}

b.d: below detection limit. 
Table 2

The specific formation/consumption rates (unit: $\mu$ mol gDCW-1 h-1) for each metabolites.

\begin{tabular}{cccc}
\hline Dilution rate (h-1) & 0.12 & 0.22 & 0.32 \\
\hline Biomass & $4550 \pm 10$ & $8134 \pm 15$ & $11469 \pm 17$ \\
Glucose (-qs) & $1460 \pm 15$ & $2429 \pm 25$ & $5049 \pm 45$ \\
Ethanol (qeth) & 0 & 0 & $3752 \pm 103$ \\
Acetate (qace) & $97 \pm 2$ & $80 \pm 4$ & $157 \pm 2$ \\
Glycerol (qgly) & $48 \pm 1$ & $33 \pm 2$ & $12988 \pm 208$ \\
CO2 (qc) & $4242 \pm 103$ & $4457 \pm 146$ & \\
\hline
\end{tabular}

\subsection{Analysis of assimilated carbon distribution}

At three different dilution rates, we did a simple analysis for the distributions of carbon assimilated by Saccharomyces cerevisiae. Based on absolute quantification of intracellular metabolites, divided into five metabolite groups (AAs, EMP, PPP, TCA, and Energy factors) and calculated the proportion of carbon content in each metabolite group. We can see that the carbon content of amino acids is much higher than other metabolite groups at three dilution rates from the figure 3 , which indicating that amino acids in yeast are the largest metabolic pool for storing carbon sources. The carbon content of the two metabolite groups (EMP and PPP) shows a certain increase with increasing in the specific 
growth rate. We analyzed the reason for this phenomenon is that, the rate for synthesis of bacteria is faster at high specific growth rate, and more intermediates (G6P, R5P, NADPH, etc.) produced by EMP and PPP are needed. At the same time, we see a significant increase in the carbon content of the TCA cycle when $\mathrm{D}=0.12 \mathrm{~h}-1$. Combined with the distributions of metabolic flux below, we know that the reason is most of the pyruvate from cytoplasm is converted to acetyl-CoA at this dilution rate, which then enters the mitochondria to participate in the TCA cycle, hence resulting in that the carbon content of TCA when $\mathrm{D}=0.12 \mathrm{~h}-1$ higher than other dilution rates.

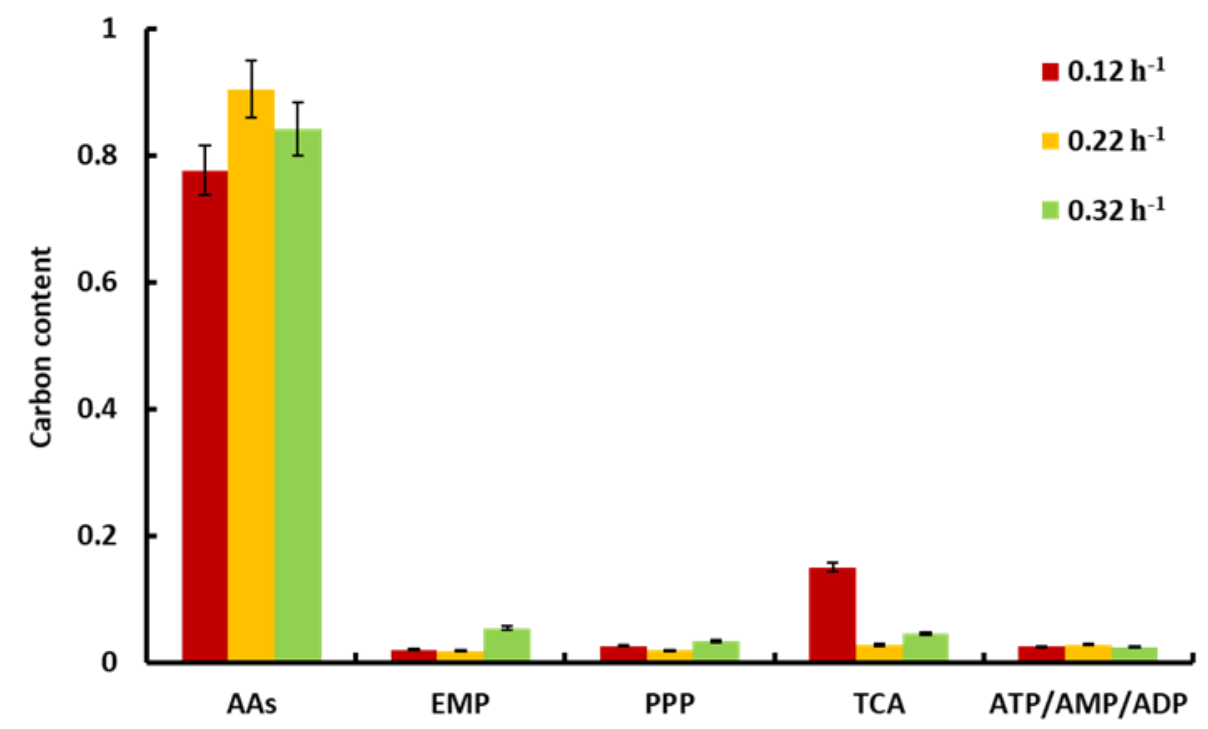

Fig 3: Distribution of carbon content at different dilution rates. Relative carbon content grouped by metabolite class with respect to the carbon consumed by $S$. cerevisiae at different growth rates. 
content.

\subsection{Free amino acid}

From the above analysis, the intracellular free amino acid is the largest metabolic pool for storing carbon sources in yeast, and the intracellular free amino acids are converted from intermediates produced in the central carbon metabolic pathway (e.g., alanine - pyruvate, glutamate $-\alpha$-ketoglutarate, and aspartate - oxaloacetate). The analysis for free amino acids may provide a novel direction for our study of metabolic metabolism, which may help us to further understand the regulation of microbial metabolism. Therefore, it is particularly important to analyze the trend of changes in the concentrations of intracellular free amino acids at different dilution rates.

As we can see from the following two figures (Fig 4 and Fig 5), the concentrations of glutamic acid, lysine, glutamine, proline, serine, aspartic acid and asparagine increase with increasing specific growth rate, where glutamine, glutamic acid and aspartic acid has increased dramatically. The concentration of tyrosine and phenylalanine decreased with increasing specific growth rate, and the concentration of phenylalanine was significantly changed when the dilution rate from 0.12 to $0.22 \mathrm{~h}-1$. In this regard, we can estimate that the precursors formed free amino acids whose concentrations show obviously changes have 
undergone significant changes under different physiological conditions. It may be related to the enzymes at protein level and the genes at transcription level, which have undergone corresponding changes, and that provides a research direction for our subsequent analysis about metabolic regulation of yeast. At the same time, we found an interesting phenomenon that the change of glutamine and glutamic are nonlinear while the change of aspartate is linear obviously.
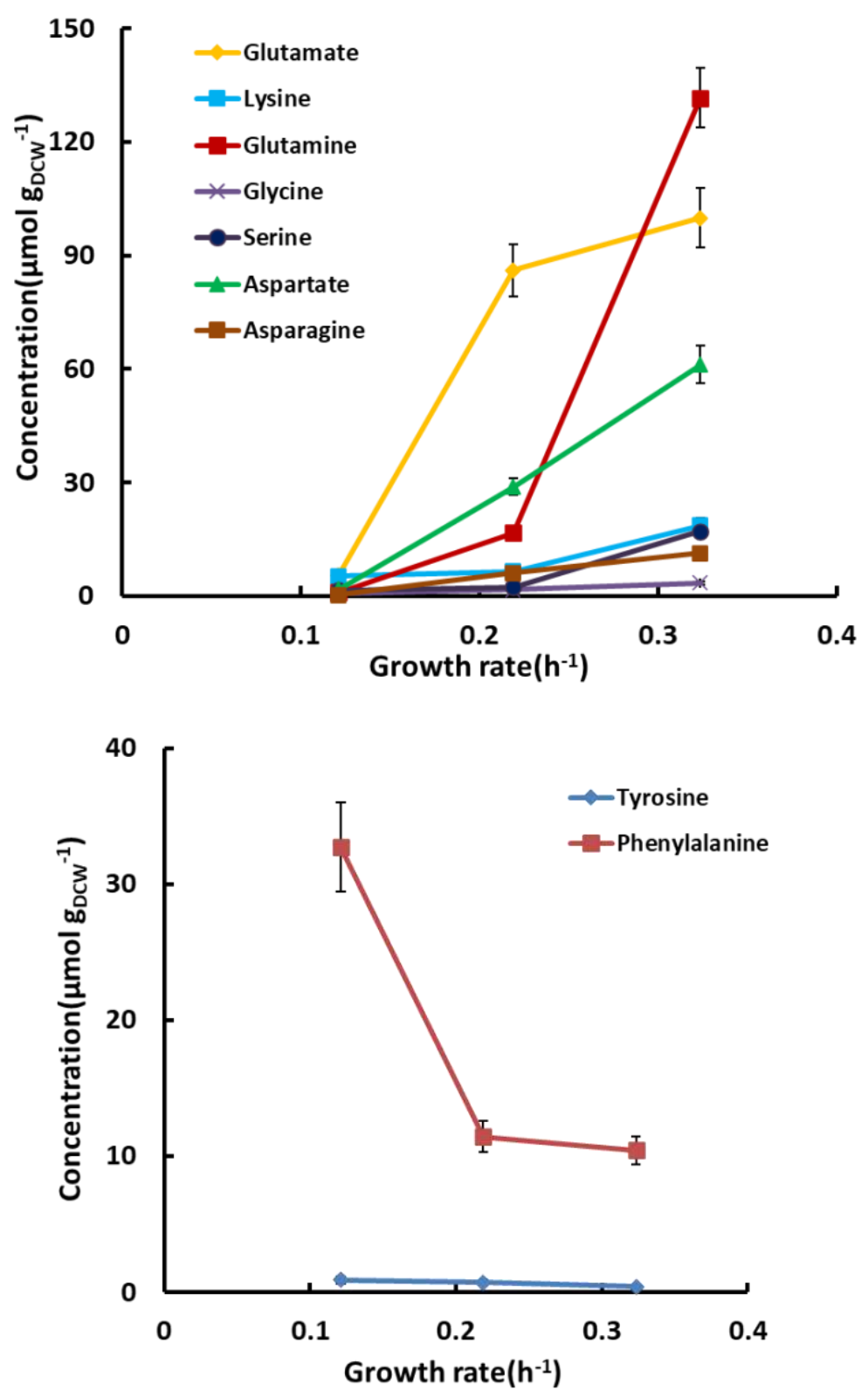
Fig 4 and Fig 5: Changes in some intracellular free amino acids at different dilution rates. The abscissa indicates the specific growth rate (h-1). The ordinate indicates the concentration $(\mu \mathrm{mol}$ gDCW-1).

\subsection{Extracellular glucose}

In the bioreactor, the enrichment of extracellular ${ }_{13} \mathrm{C}$-glucose is changes over time after the substrate is switched to ${ }_{13} \mathrm{C}$ labeled glucose. The carbon enrichment of extracellular glucose in time is influenced by: the ${ }_{13} \mathrm{C}$ feed flow rate, the specific glucose uptake rate, and the residual ${ }_{12} \mathrm{C}$-glucose concentration. About this, we made a simple analysis for the process that how the carbon enrichment of extracellular glucose is changing. We calculated the corresponding turnover time $(\tau)$ at three dilution rates $\mathrm{D}=0.12,0.22,0.32 \mathrm{~h}-1$, which is 257,155 , and $400 \mathrm{sec}$, respectively. Accordingly, the fastest extracellular glucose enrichment was observed at $\mathrm{D}=0.22 \mathrm{~h}-1$ and that is consistent with the findings of others (Suarez-Mendez et al. 2016), while the slowest was at $D=0.32 \mathrm{~h}-1$. For the reason why the slowest extracellular ${ }_{13} \mathrm{C}$ glucose enrichment was at $\mathrm{D}=0.32 \mathrm{~h}-1$, we believe the main reason is that the extracellular residual glucose concentration is too high at this dilution rate. In addition, we also studied the relationship between extracellular ${ }_{13} \mathrm{C}$ glucose enrichment and $\tau$, it shown in the figure 7 . We found that the trend of extracellular ${ }_{13} \mathrm{C}$ glucose enrichment and $\tau$ was basically the same for three different specific growth rates. After $5 \tau$, all the carbon enrichment reached $80 \%$, and it also approached stability. 


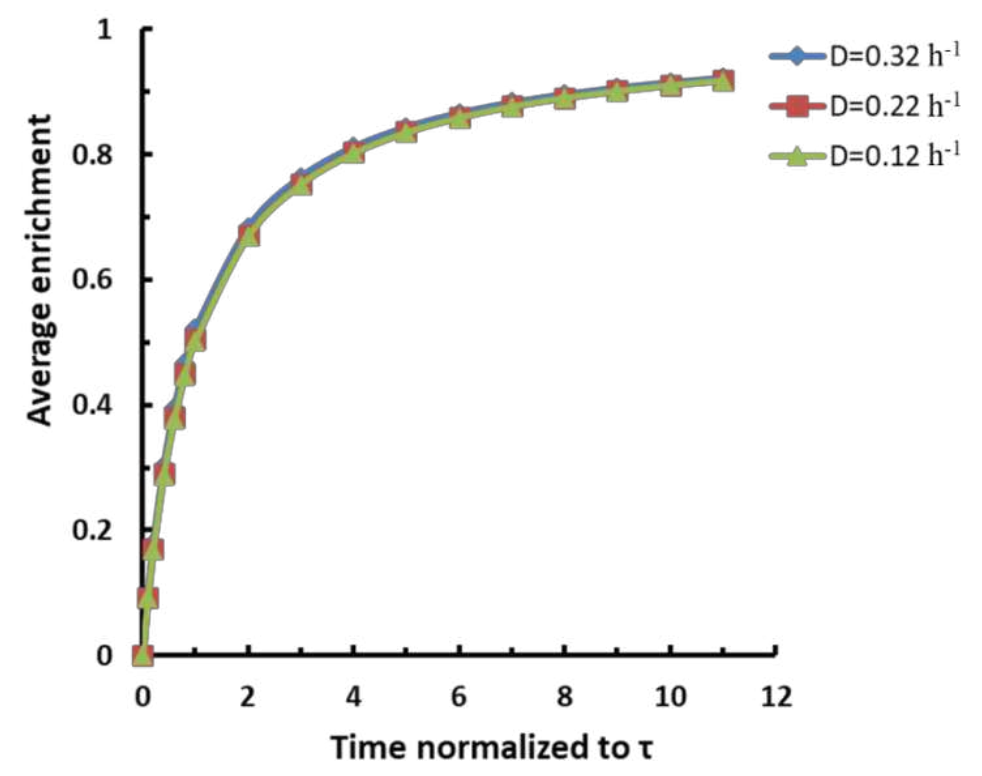

Fig 7: Carbon enrichment of extracellular glucose. Dynamics of ${ }_{13} \mathrm{C}$ enrichment at different dilution rates as function of time normalized by the turnover time of extracellular glucose pool (experimental time/ $\tau$ ). The abscissa represents time and the time was normalized to $\tau$. The ordinate indicates average enrichment of extracellular ${ }_{13} \mathrm{C}$-glucose.

\subsection{Carbon flux distribution at the G6P branch}

Flux maps at three different dilution rates are shown in supplementary material 2 . We analyze the distribution of the metabolic flux on the G6P node (see Table 3). Most of the carbon influx at the G6P node was directed to the EMP pathway for these three different dilution rates. Especially at a high specific growth rate $(D=0.32 h-1)$, and it reached the maximum value which is $77.31 \%$ (the glucose uptake rate is set to 1). That is consistent with the results of previous experiments (Suarez-Mendez et al. 2016), and it turns out that the excess carbons were generated pyruvate via the EMP pathway, and then converted to ethanol 
subsequently. Therefore, ethanol can be detected extracellularly at a high specific growth rate. At the same time, we found that the flux ratio of the PP pathway fluctuated between $10.44 \%$ and $14.68 \%$ with no significant changes. We compared the proportion of flux on PP pathway with other's works. The similarity is that the PP pathway ratio is $14.7 \%$ when $\mathrm{D}=0.2$ $\mathrm{h}-1$, and the difference is that their value is $3.8 \%$ while our value is $14.15 \%$ when $\mathrm{D}=0.1 \mathrm{~h}-1$ (Suarez-Mendez et al. 2016). We suspect that there may be differences in the species, culture system, or labeling substrate.

Table 3

Carbon flux distribution at the G6P node (all values are relative and glucose uptake rate set to 1).

\begin{tabular}{cccc}
\hline Dilution rate (h-1) & 0.12 & 0.22 & 0.32 \\
\hline PPP split ratio & 0.1415 & 0.1468 & 0.1044 \\
EMP split ratio & 0.7305 & 0.6786 & 0.7731 \\
Biomass split ratio & 0.1280 & 0.1747 & 0.1243 \\
\hline
\end{tabular}

\subsection{TCA cycle}

We list the flux proportions of the TCA cycle at three different physiological states (see figure 8 ). It can be found that the flux ratio of TCA cycle gradually decreases as the dilution rate increases, and the trends of decrease are significant. Therefore, it can be judged that the yeast undergoes metabolic migration due to its own regulation during the transitions of dilution rate, and some carbon do not enter the 
mitochondria to participate in the TCA cycle. From figure we can see that most of the carbon flux were involved in the TCA cycle when $\mathrm{D}=0.12$ $\mathrm{h}-1$ at these three dilution rates. However, it was the opposite when $\mathrm{D}=$ $0.32 \mathrm{~h}-1$.

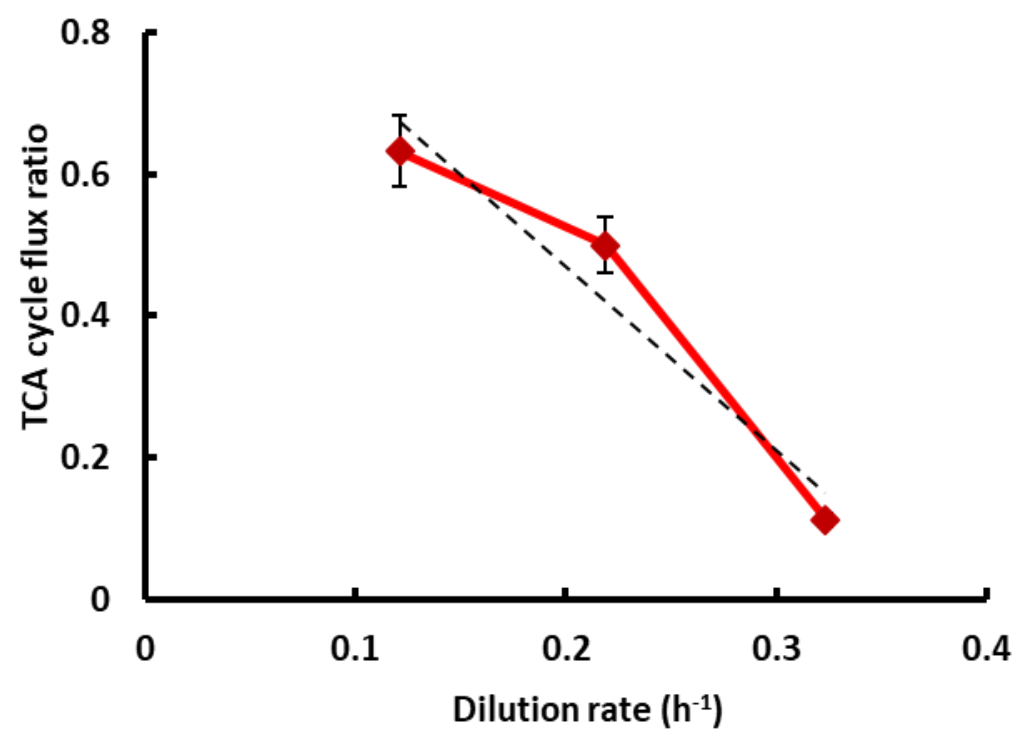

Fig 8: Carbon flux distribution at TCA cycle and all values are relative and glucose uptake rate set to 1 .

\subsection{The yield of biomass and by-product}

In our research, we found an interesting phenomenon as we put the yield of biomass and the relative flux of EMP/PP pathway at these three dilution rates on one graph (see Figure 9). And we will find that the yield of biomass increased and then decreased with the dilution rate increasing. The reason why the biomass yield was decrease is that more carbons are converted to ethanol, not biomass at higher dilution rates. At the same time, we also found that the change of the relative flux on PP pathway is 
consistent with the change of the yield of biomass. However, it is the opposite for EMP, and the result about that is worthy of further research and analysis. The PP pathway mainly provides essential precursors for the synthesis of biomass (e.g., R5P, X5P, NADPH, etc.), therefore, the yield of biomass is positively correlated with the relative flux of PP pathway. And the EMP provides the precursor substance pyruvate, were converted into extracellular by-product (e.g., ethanol, acetate). So, the yield of biomass is inversely correlated with the EMP relative flux.

After analyzing the biomass yield, then we analyzed the yield of extracellular by-products (see Figure 10). From the figure we can see that the yield of extracellular products acetic acid and glycerol did not change significantly at these three dilution rates. Therefore, it can be inferred that the metabolic flux (relative flux) of producing acetate and glycerol did not change significantly during the changes on dilution rate. There is no ethanol production at the first two low dilution rates, but the ethanol yield rises sharply at high dilution rates $(\mathrm{D}=0.32 \mathrm{~h}-1)$. And this provides a research direction for the "metabolic migration" we mentioned earlier. Combining the yield of biomass and the yield of by-products, we found that a large amount of carbons enter the EMP pathway which further converted into ethanol, then resulting in the declines of biomass yield and PPP flux (relative flux) under the high specific growth rate $(D=0.32 \mathrm{~h}-1)$. 

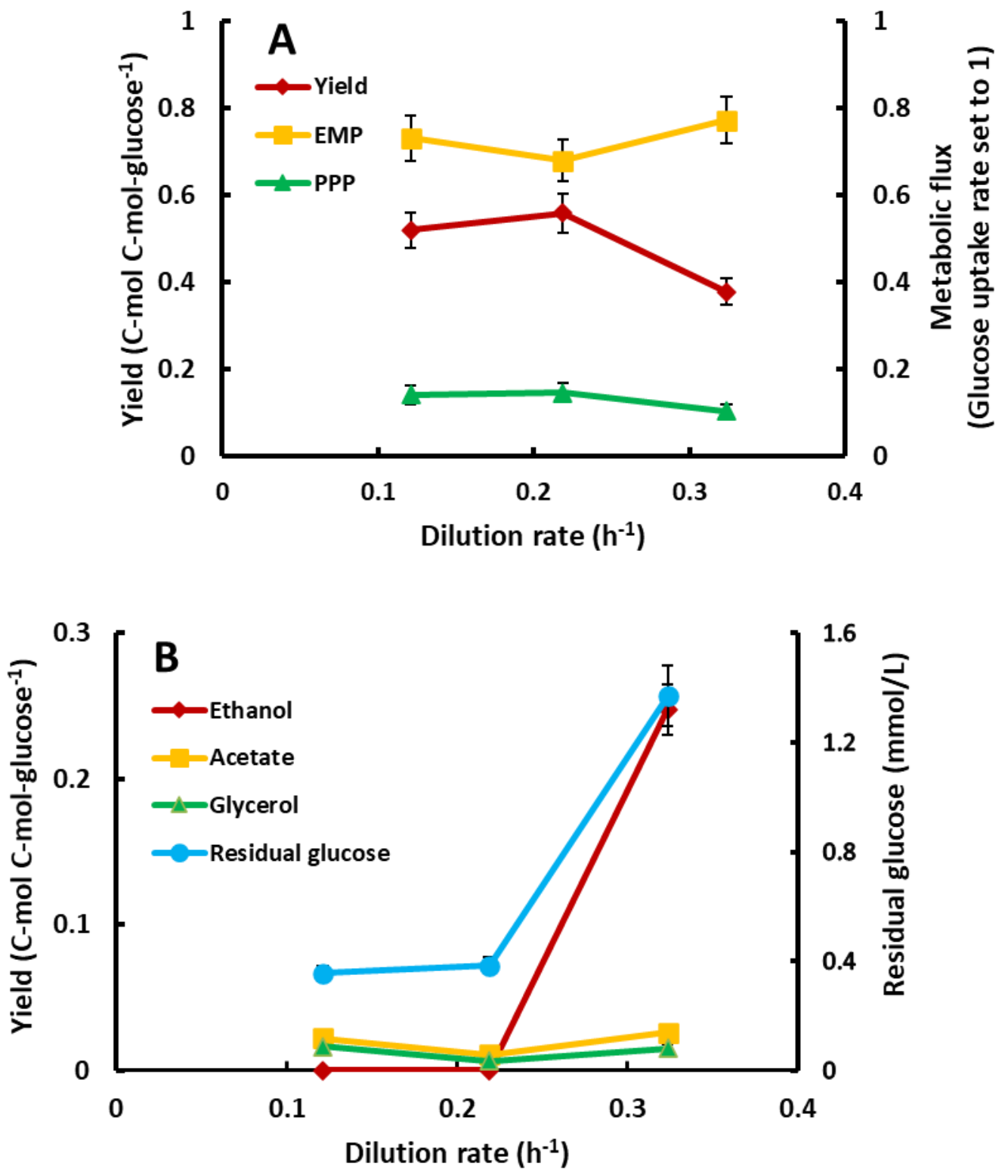

Fig 9: The relationship between the yield of biomass and the relative flux of EMP/PPP pathway at these three dilution rates. The abscissa indicates the dilution rate (h-1), the main ordinate indicates the biomass yield (C-mol C-mol-glucose-1), secondary ordinate indicates the relative flux of EMP/PPP pathway (when glucose uptake rate set to 1). Fig 10: The relationship between the yield of by-products and dilution rates. The abscissa indicates the dilution rate (h-1), the ordinate indicates the yield of by-products (C-mol C-mol-glucose-1).

\subsection{ATP balance and NADPH balance}


In addition, we also analyzed the balance of ATP (see Fig 11) and NADPH (see Fig 12). For the generation of ATP, it is mainly derived from substrate level phosphorylation and electron transport chain, and the electron transport chain is based on $\mathrm{P} / \mathrm{O}=1$ (Rigoulet et al. 1989). The consumption of ATP is mainly used to maintain the growth of the yeast, and it was estimated by using a constant of $69.2 \mathrm{mmol} \mathrm{ATP} / \mathrm{gDCw}_{\text {DForster }}$
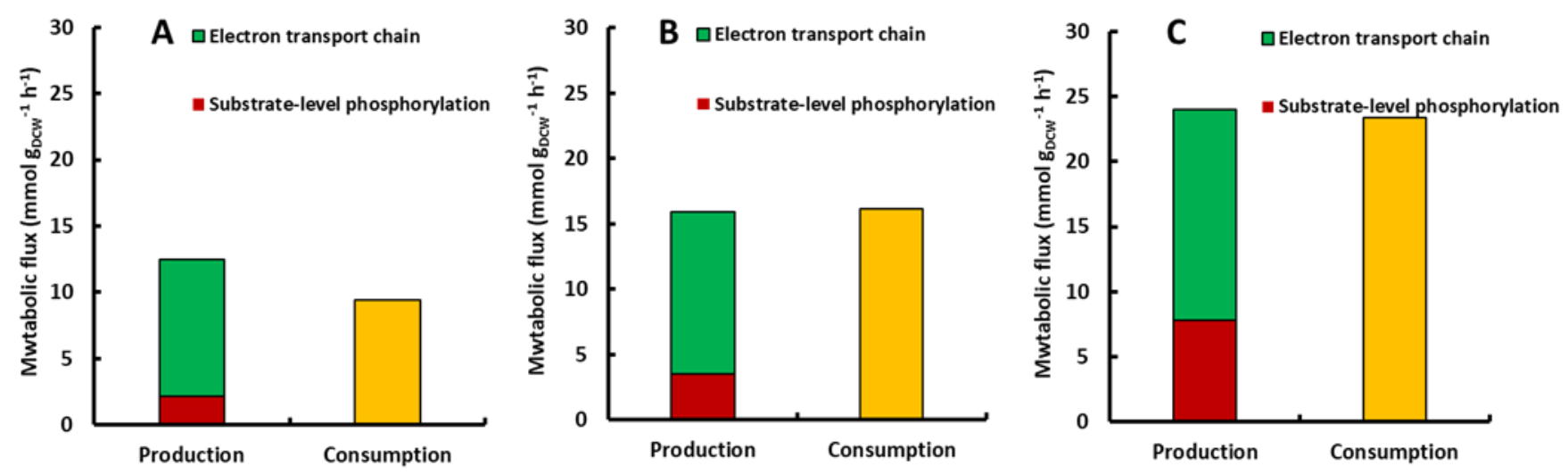

et al. 2003). It can be seen that most of the ATP is produced by reactions on the electron transport chain at different dilution rates. And the ratio of ATP produced by substrate-level phosphorylation are $17.08 \%, 22.23 \%$, $32.43 \%$ when $\mathrm{D}=0.12,0.22,0.32 \mathrm{~h}-1$ respectively, it is obvious that the ratio of ATP produced by substrate-level phosphorylation increase gradually. Meantime, the rate of ATP production increases gradually as the dilution rate increases. The reason is that more carbons enter the yeast to participate in the reaction to provide ATP with the glucose consumption rate increases. ATP balance was closed strictly at $\mathrm{D}=0.22$ and $0.32 \mathrm{~h}-1$, but the balance was not closed at $\mathrm{D}=0.12 \mathrm{~h}-1$. 
Fig 11: Production and consumption of ATP. The production and consumption rates (mmol gDCw-1 h-1) of ATP were estimated using the metabolic flux distribution under three dilution rates. (A). $D=0.12$ h-1 (B). $D=0.22$ h-1 (C). $\mathrm{D}=0.32 \mathrm{~h}-1$

The production of NADPH mainly comes from the reactions on the PPP pathway. And the consumption of NADPH is mainly used for the synthesis of biomass, such as synthetic nucleotides, amino acids, lipids, etc. We used a constant of $9.31 \mathrm{mmol} \mathrm{NADPH} / \mathrm{gDCw}$ to characterize the consumption of NADPH (Bruinenberg et al. 1986). The rate of NADPH
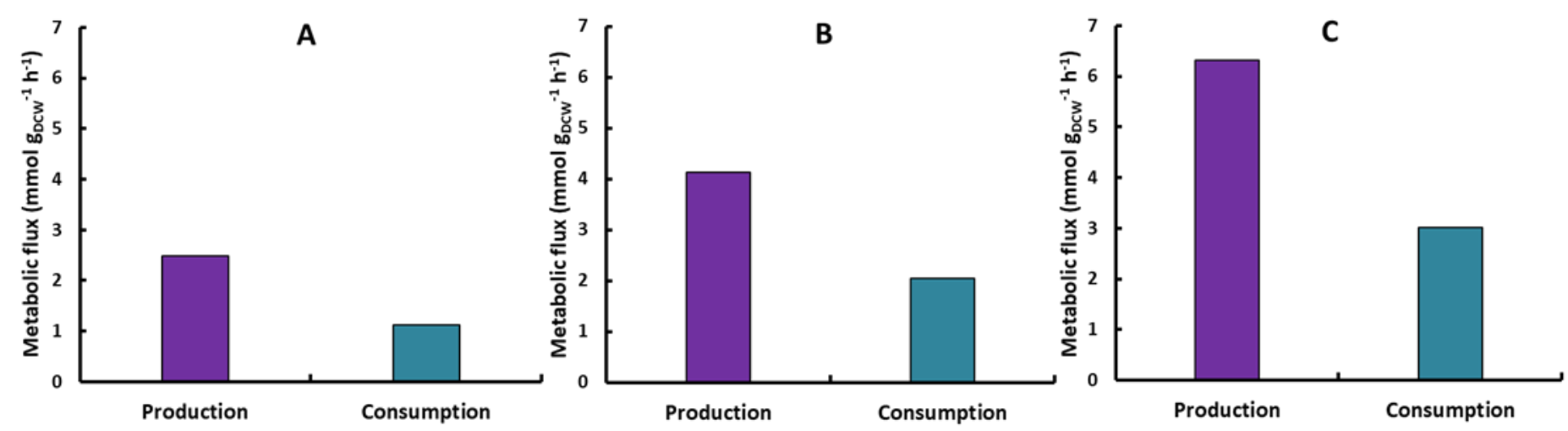

formation increases with increasing dilution rates due to an increase in flux (absolute flux) over the PPP pathway. However, at three dilution rates, the balance of the NADPH production and consumption rates, estimated from metabolic flux distribution, was not closed. It has been explained that this is because some of the NADPH is transported into the mitochondria and then participate in the reactions on the electron transport chain to generate ATP (Sollner et al. 2009). 
Fig 12: Production and consumption of NADPH. The production and consumption rates (mmol gDCw-1 h-1) of NADPH were estimated using the metabolic flux distribution under three dilution rates. (A). D=0.12 h-1 (B). $\mathrm{D}=0.22 \mathrm{~h}-1(\mathrm{C}) . \mathrm{D}=0.32 \mathrm{~h}-1$

\section{Discussion}

It shows that there is a significant change of the flux distribution of intracellular central carbon metabolic under different physiological conditions by using the ${ }_{13} \mathrm{C}$ metabolic flux analysis of Saccharomyces cerevisiae. And the flux distributions were closely related to the environment surrounding bacteria. In our research, we think it was accompanied with metabolic migration when $S$. cerevisiae suffer from low specific growth rate to high specific growth rate. Our results indicate there was a sharp rise in ethanol yield and a sharp drop in TCA flux (relative flux), it is consistent with results of previous research (Frick and Wittmann 2005). Moreover, we also found that the bacterial yield has a certain relationship with the metabolic flux (relative value) on the EMP and PP pathways.

i. Our works show that the changes in physiological status have a significant impact on the flux distribution of EMP, PPP, and TCA (see supplementary material 2). The trends of flux on the PPP against with the EMP, the two pathways have a certain relationship with biomass yield. As analyzed above, the biomass 
yield was negatively correlated with the flux (relative flux) of the EMP and positively correlated with the flux (relative flux) of the PPP, and that was not reported previously. We think it is related to the functions of various metabolic pathways in yeast. We guess the reason is that PPP mainly provides essential precursors for the synthesis of biomass while the EMP provides the unique precursor substance pyruvate converted into extracellular by-product (e.g., ethanol, acetate) during the process. For the TCA cycle, the metabolic flux (relative flux) shows a significantly decreasing trend as the dilution rate increases. At the same time, we observed that the Crabtree effect occurred during the process of increasing dilution rate. It is the yield of ethanol has increased dramatically, which provides a direction for us to study the metabolic migration. For the G6P node, the previous study about the enzyme activity of Saccharomyces cerevisiae under chemostat culture conditions showed that the activity of G6P dehydrogenase was slightly increased with $\mathrm{D}=0.1 \mathrm{~h}-1$ increases to $0.4 \mathrm{~h}-1$ (Serra, Strehaiano, and Taillandier 2003). In our work, at three dilution rates $(\mathrm{D}=0.12,0.22,0.32$ h-1), the rates of reaction catalyzed by G6P dehydrogenase was $0.0574,0.0955,0.1464 \mu \mathrm{mol} / \mathrm{g} \mathrm{DCW} / \mathrm{sec}$, respectively. This is consistent with that reported by Seera (2003). G6P 
dehydrogenase is the first and key enzyme in PP pathway. We found that the flux (relative value) of PPP calculated at a low specific growth rate by others is only a little higher than the high specific growth rate (Gombert et al. 2001), which is close to our results. At the same time, there are cases that the flux is much higher (Frick and Wittmann 2005). We suspect that the reason for this phenomenon maybe the differences in culture systems or strains (other's strain is Penicillium chrysogenum).

ii. In Saccharomyces cerevisiae, pyruvate decarboxylase is a key enzyme that catalyzes certain reactions produced precursor substances for the TCA cycle. Pyruvate is catalyzed by pyruvate decarboxylase to form acetaldehyde which is catalyzed by acetaldehyde dehydrogenase to form acetate. And pyruvate catalyzed by acetyl-CoA synthetase to form acetyl-CoA which enters the mitochondria and participates in the TCA cycle. We found that the flux distribution around the pyruvate node has changed significantly under these three different physiological states. Especially, a large amount of pyruvate is converted to acetaldehyde and then participates in subsequent reactions at high dilution rate. This indicates that a large amount of pyruvate is still involved in the fermentation reaction when S. cerevisiae is in the state of producing ethanol, and it refuting the view that 
only a small proportion of pyruvate is involved (Lei, Rotboll, and Jorgensen 2001). Rather, it supports the view that the flux distribution around the pyruvate node is related to "Crabtree effect" of Saccharomyces cerevisiae (Postma et al. 1989). The flux flowed into acetyl-CoA from pyruvate has been high, whether at low specific growth rates or at high specific growth rates. And it indicates that the activity of pyruvate decarboxylase has been in an excellent state all the time. Our work also shows that the concentration of pyruvate increases with increasing dilution rate, which is consistent with the previously reported results (Postma et al. 1989). We suspect that the reason may be that some pyruvate in the mitochondria is transferred to the cytoplasm for the synthesis of alanine.

iii. By combining the relative values (glucose uptake rate set to 1) of the metabolic flux distribution acquired from OpenMebius and the rate of glucose consumption, we can obtain the absolute rate of the reaction in the central carbon metabolic of Saccharomyces cerevisiae at different growth rate. From the perspective of the absolute value of metabolic flux, we can further understand the relationship between some complex reactions in central carbon metabolic. And the specific consumption rate of glucose increases with the increase of specific growth rate, increases by 
nearly 4 times (the specific consumption rate of glucose is $0.4056,0.6748,1.4026 \mu \mathrm{mol}$ gDCW-1 sec-1 under $\mathrm{D}=0.12,0.22$, $0.32 \mathrm{~h}-1$, respectively). Therefore, how the increased carbon sources are distributed in the central carbon metabolic is worth analysis. At these three dilution rates, the metabolic flux (absolute value) of the reaction catalyzed by pyruvate decarboxylase, acetaldehyde dehydrogenase, and acetyl-CoA synthetase is shown below (see Fig 13). As we can see from the figure, the metabolic flux associated with pyruvate decarboxylase increases sharply with increasing specific growth rate, while the metabolic flux associated with acetaldehyde dehydrogenase and acetyl-CoA synthetase keep steady nearly. Further, there was no significant change in the metabolic flux associated with acetyl-CoA synthetase at different dilution rates, and it indicates that the acetyl-CoA synthetase has reached a state of work saturation regardless of the low dilution rate or the high dilution rate. Therefore, the accumulated acetate is discharged from the cells, so that we can detect the by-product acetate extracellularly. Moreover, the rate of acetate production is gradually increased, and the specific production rate of acetate is $0.0271,0.0450,0.1093 \mu \mathrm{mol}$ gDCW-1 $\mathrm{h}-1$ at $\mathrm{D}=0.12,0.22,0.32$ $\mathrm{h}-1$, respectively, which is closely related to that acetyl-CoA 
synthetase has reached a state of work saturation. Aldehyde dehydrogenase and acetyl-CoA synthetase are the key enzymes for the synthesis of acetyl-CoA, which can further synthesize the amino acids and lipids required for the growth of the yeast. Studies have shown that the activity of acetaldehyde dehydrogenase and acetyl-CoA synthetase decreases in the in-vitro enzyme activity experiment when the metabolic state changes from respiration to fermentation (Serra, Strehaiano, and Taillandier 2003), this is also consistent with our results. These enzymes catalyze certain key reactions and have great significance for guiding the transformation of strains.

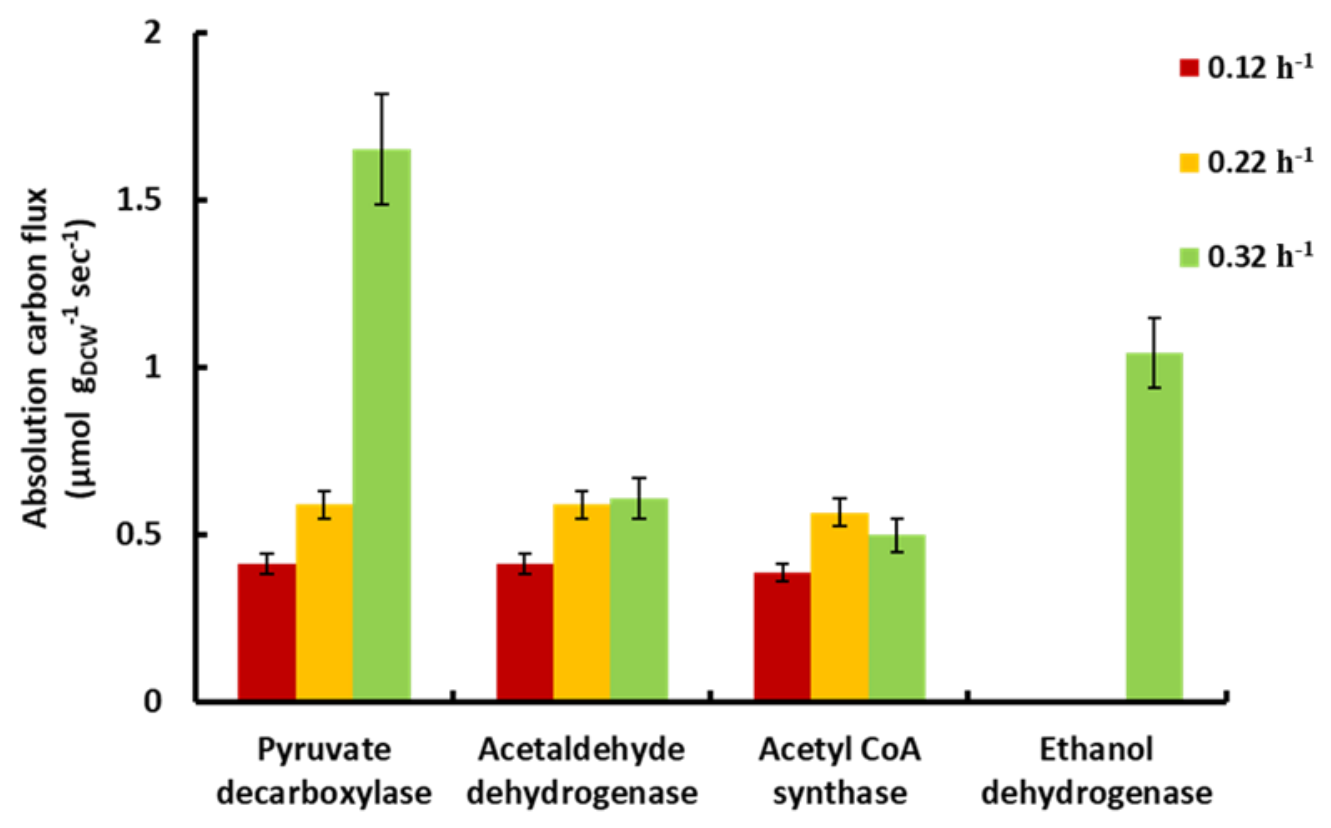

Fig 13: The metabolic flux (absolute value) of the reaction catalyzed by pyruvate decarboxylase, 
three different enzymes. The ordinate indicates the reaction flux (absolute value) ( $\mu$ mol gDCW-1 h-1)

catalyzed by the corresponding enzyme.

\section{Conclusions}

Crabtree effect is well known for Saccharomyces cerevisiae, and we also encountered this imagination in our work. However, its triggering mechanisms are still unknown. In general, the changes of environmental influences the metabolism-related regulatory factors which further affects the metabolic distributions of the entire central carbon metabolic in $S$. cerevisiae. When the physiological state changes from a low specific growth rate to a high specific growth rate, the metabolic regulation of Saccharomyces cerevisiae will change accordingly, such as enzyme activity, stress resistance or self-morphology, etc. These corresponding changes are controlled by complex transcriptional or post-transcriptional systems (Daran-Lapujade et al. 2004). One of the most important factors affecting the metabolic mechanism of Saccharomyces cerevisiae in different environments is the concentration of glucose in the medium (Rolland, Winderickx, and Thevelein 2002). It has been proven in our work, the concentration of glucose in the medium increased with increasing specific growth rate (when $\mathrm{D}=0.12,0.22,0.32-1$, glucose concentration was $0.3567,0.3839,1.3706 \mathrm{mmol} / \mathrm{L})$. Especially at $\mathrm{D}=0.32$ $\mathrm{h}_{-1}$, the glucose concentration increased nearly 4 times. At the same time, 
the distribution of intracellular metabolic flux has also changed a lot. It has been previously reported that the main signal triggering changes in intracellular metabolic flux may be the glucose suppression system (Frick and Wittmann 2005). As the specific growth rate increases, the metabolic flux (relative value) of the TCA cycle is reduced. It may be that the glucose suppression system affects some genes in the TCA cycle at the transcriptional level (Schuller 2003). Similarly, the specific rate of glucose consumption and the metabolic flux of EMP pathways increase significantly, and may also affect related genes at the transcriptional level (Carlson 1999). Recently, studies on metabolic distributions and transcriptional level regulation have shown that the intracellular mechanism of Saccharomyces cerevisiae may also be regulated by post-transcriptional levels (Daran-Lapujade et al. 2004). Through the above analysis, it can be known that the metabolic flux characterizes the results combined with the metabolic regulation of microorganisms at different levels. Therefore, metabolic flux is a quantitative description for the intracellular metabolic state. In our review, we cannot use the single factor such as metabolic flux distribution to analyze or explain the changes of the metabolic regulation mechanism of Saccharomyces cerevisiae under different environments. However, the absolute quantification of the metabolic distribution of $S$. cerevisiae in different environments helps us understand its regulatory mechanisms at the level 
of metabolic flux. If combined with metabolic flux, proteome, transcriptome and other omics, it will help us to have a global understanding of the regulation of microbial metabolism.

Abbreviations: CDW, cell dry weight; $13 \mathrm{C}-\mathrm{MFA}$ : $13 \mathrm{C}$ metabolic flux analysis; INST-13C-MFA, Isotopically nonstationary metabolic flux analysis; CCM, central carbon metabolism; AcCoA, acetyl-CoA synthase; EMP, embden meyerh of pathway; TCA, tricarboxylic acid cycle; PPP, pentose phosphate pathway; AMP, adenosine-monophosphate; ADP, adenosine-diphosphate; ATP, adenosine-triphosphate; G6P, glucose-6-phosphate; $\quad$ F6P, fructose-6-phosphate; $\quad$ 6PG, 6-phosphogluconate; S7P, eedoheptulose-7-phosphate; E4P, erythrose-4-phosphate; FBP, fructose-1,6-bisphosphate; GAP, glyceraldehyde-3-phosphate; PEP, phosphoenolpyruvate; Iso-CIT, isocitrate; AKG, $\alpha$-ketoglutarate; SUC, succinate; FUM, fumarate; MAL, L-malate; NADPH, Nicotinamide Adenine Dinucleotide Phosphate.

\section{Declarations}

- Ethics approval and consent to participate

Not applicable

- Consent for publication

Not applicable 
- Availability of data and materials

The datasets used and/or analyzed during the current study are available from the corresponding author on reasonable request.

- Competing interests

The authors declare that they have no competing interests.

- Funding

This work was supported by the National Natural Science Foundation of China [Grant Numbers 21506052, 21776082]; and the Fundamental Research Funds for the Central Universities [Grant Number 22221818014]; and the 111 Project [Grant Number B18022].

- Authors' contributions

HL analyzed and interpreted the change of the ATP paradox and the $\mathrm{NAD}+/ \mathrm{NADH}$ ratio. WS and PL designed and implemented U-13C labeled cell extracts. HL and MC extracted the intracellular metabolites. HL and JYX were major contributors in writing the manuscript. All authors read and approved the final manuscript.

- Acknowledgements

Not applicable.

- Authors' information

Huan L: East China university of science and technology, State Key Laboratory of Bioreactor Engineering.

Min C: East China university of science and technology, State Key 


\section{Laboratory of Bioreactor Engineering.}

Shuai W: East China university of science and technology, State Key Laboratory of Bioreactor Engineering.

Peng L: East China university of science and technology, State Key Laboratory of Bioreactor Engineering.

JY Xia: East China university of science and technology, State Key Laboratory of Bioreactor Engineering.

\section{References}

Antoniewicz, M. R., J. K. Kelleher, and G. Stephanopoulos. 2007. 'Elementary metabolite units (EMU): a novel framework for modeling isotopic distributions', Metab Eng, 9: 68-86.

Bartek, T., B. Blombach, S. Lang, B. J. Eikmanns, W. Wiechert, M. Oldiges, K. Noh, and S. Noack. 2011. 'Comparative 13C metabolic flux analysis of pyruvate dehydrogenase complex-deficient, L-valine-producing Corynebacterium glutamicum', Appl Environ Microbiol, 77: 6644-52.

Becker, J., A. Lange, J. Fabarius, and C. Wittmann. 2015. 'Top value platform chemicals: bio-based production of organic acids', Curr Opin Biotechnol, 36: 168-75.

Becker, J., and C. Wittmann. 2012. 'Bio-based production of chemicals, materials and fuels -Corynebacterium glutamicum as versatile cell factory', Curr Opin Biotechnol, 23: 631-40.

- 2016. 'Systems metabolic engineering of Escherichia coli for the heterologous production of high value molecules-a veteran at new shores', Curr Opin Biotechnol, 42: 178-88.

Blank, L. M., F. Lehmbeck, and U. Sauer. 2005. 'Metabolic-flux and network analysis in fourteen hemiascomycetous yeasts', FEMS Yeast Res, 5: 545-58.

Boghigian, B. A., G. Seth, R. Kiss, and B. A. Pfeifer. 2010. 'Metabolic flux analysis and pharmaceutical production', Metab Eng, 12: 81-95.

Bruinenberg, P. M., G. W. Waslander, J. P. van Dijken, and W. A. Scheffers. 1986. 'A comparative radiorespirometric study of glucose metabolism in yeasts', Yeast, 2: 117-21.

Burk, M. J., and S. Van Dien. 2016. 'Biotechnology for Chemical Production: Challenges and Opportunities', Trends in Biotechnology, 34: 187-90.

Canelas, A. B., C. Ras, A. ten Pierick, W. M. van Gulik, and J. J. Heijnen. 2011. 'An in vivo data-driven framework for classification and quantification of enzyme kinetics and determination of apparent thermodynamic data', Metab Eng, 13: 294-306.

Canelas, A. B., A. ten Pierick, C. Ras, R. M. Seifar, J. C. van Dam, W. M. van Gulik, and J. J. Heijnen. 2009. 'Quantitative evaluation of intracellular metabolite extraction techniques for yeast metabolomics', Anal Chem, 81: 7379-89.

Carlson, M. 1999. 'Glucose repression in yeast', Curr Opin Microbiol, 2: 202-7. 
Castrillo, J. I., L. A. Zeef, D. C. Hoyle, N. Zhang, A. Hayes, D. C. Gardner, M. J. Cornell, J. Petty, L. Hakes, L. Wardleworth, B. Rash, M. Brown, W. B. Dunn, D. Broadhurst, K. O'Donoghue, S. S. Hester, T. P. Dunkley, S. R. Hart, N. Swainston, P. Li, S. J. Gaskell, N. W. Paton, K. S. Lilley, D. B. Kell, and S. G. Oliver. 2007. 'Growth control of the eukaryote cell: a systems biology study in yeast', J Biol, 6: 4.

Cipollina, C., A. ten Pierick, A. B. Canelas, R. M. Seifar, A. J. van Maris, J. C. van Dam, and J. J. Heijnen. 2009. 'A comprehensive method for the quantification of the non-oxidative pentose phosphate pathway intermediates in Saccharomyces cerevisiae by GC-IDMS', $J$ Chromatogr B Analyt Technol Biomed Life Sci, 877: 3231-6.

Daran-Lapujade, P., M. L. Jansen, J. M. Daran, W. van Gulik, J. H. de Winde, and J. T. Pronk. 2004. 'Role of transcriptional regulation in controlling fluxes in central carbon metabolism of Saccharomyces cerevisiae. A chemostat culture study', J Biol Chem, 279: 9125-38.

De Deken, R. H. 1966. 'The Crabtree effect: a regulatory system in yeast', J Gen Microbiol, 44: 149-56. de Jonge, L. P., N. A. Buijs, A. ten Pierick, A. Deshmukh, Z. Zhao, J. A. Kiel, J. J. Heijnen, and W. M. van Gulik. 2011. 'Scale-down of penicillin production in Penicillium chrysogenum', Biotechnology Journal, 6: 944-58.

Forster, J., I. Famili, P. Fu, B. O. Palsson, and J. Nielsen. 2003. 'Genome-scale reconstruction of the Saccharomyces cerevisiae metabolic network', Genome Research, 13: 244-53.

Frick, O., and C. Wittmann. 2005. 'Characterization of the metabolic shift between oxidative and fermentative growth in Saccharomyces cerevisiae by comparative 13C flux analysis', Microb Cell Fact, 4: 30.

Gombert, A. K., M. M. dos Santos, B. Christensen, and J. Nielsen. 2001. 'Network identification and flux quantification in the central metabolism of Saccharomyces cerevisiae under different conditions of glucose repression', Journal of Bacteriology, 183: 1441-51.

Hackett, S. R., V. R. Zanotelli, W. Xu, J. Goya, J. O. Park, D. H. Perlman, P. A. Gibney, D. Botstein, J. D. Storey, and J. D. Rabinowitz. 2016. 'Systems-level analysis of mechanisms regulating yeast metabolic flux', Science, 354.

Hashem, M., and S. M. I. Darwish. 2010. 'Production of bioethanol and associated by-products from potato starch residue stream by Saccharomyces cerevisiae', Biomass \& Bioenergy, 34: 953-59.

Kajihata, S., C. Furusawa, F. Matsuda, and H. Shimizu. 2014. 'OpenMebius: an open source software for isotopically nonstationary 13C-based metabolic flux analysis', Biomed Res Int, 2014: 627014.

Lehnen, M., B. E. Ebert, and L. M. Blank. 2017. 'A comprehensive evaluation of constraining amino acid biosynthesis in compartmented models for metabolic flux analysis', Metab Eng Commun, 5: 34-44.

Lei, F., M. Rotboll, and S. B. Jorgensen. 2001. 'A biochemically structured model for Saccharomyces cerevisiae', J Biotechnol, 88: 205-21.

Long, C. P., and M. R. Antoniewicz. 2014. 'Metabolic flux analysis of Escherichia coli knockouts: lessons from the Keio collection and future outlook', Curr Opin Biotechnol, 28: 127-33.

Mashego, M. R., W. M. van Gulik, J. L. Vinke, D. Visser, and J. J. Heijnen. 2006. 'In vivo kinetics with rapid perturbation experiments in Saccharomyces cerevisiae using a second-generation BioScope', Metab Eng, 8: 370-83.

Mashego, M. R., L. Wu, J. C. Van Dam, C. Ras, J. L. Vinke, W. A. Van Winden, W. M. Van Gulik, and J. J. Heijnen. 2004. 'MIRACLE: mass isotopomer ratio analysis of U-13C-labeled extracts. A 
new method for accurate quantification of changes in concentrations of intracellular metabolites', Biotechnol Bioeng, 85: 620-8.

Pereira, M. D., E. C. Eleutherio, and A. D. Panek. 2001. 'Acquisition of tolerance against oxidative damage in Saccharomyces cerevisiae', BMC Microbiol, 1: 11.

Petersen, S., A. A. de Graaf, L. Eggeling, M. Mollney, W. Wiechert, and H. Sahm. 2000. 'In vivo quantification of parallel and bidirectional fluxes in the anaplerosis of Corynebacterium glutamicum', J Biol Chem, 275: 35932-41.

Postma, E., C. Verduyn, W. A. Scheffers, and J. P. Van Dijken. 1989. 'Enzymic analysis of the crabtree effect in glucose-limited chemostat cultures of Saccharomyces cerevisiae', Appl Environ Microbiol, 55: 468-77.

Quek, L. E., C. Wittmann, L. K. Nielsen, and J. O. Kromer. 2009. 'OpenFLUX: efficient modelling software for 13C-based metabolic flux analysis', Microb Cell Fact, 8: 25.

Rigoulet, M., R. Ouhabi, X. Leverve, F. Putod-Paramelle, and B. Guerin. 1989. 'Almitrine, a new kind of energy-transduction inhibitor acting on mitochondrial ATP synthase', Biochim Biophys Acta, 975: $325-9$.

Rolland, F., J. Winderickx, and J. M. Thevelein. 2002. 'Glucose-sensing and -signalling mechanisms in yeast', FEMS Yeast Res, 2: 183-201.

Sauer, U. 2006. 'Metabolic networks in motion: 13C-based flux analysis', Mol Syst Biol, 2: 62.

Schuller, H. J. 2003. 'Transcriptional control of nonfermentative metabolism in the yeast Saccharomyces cerevisiae', Curr Genet, 43: 139-60.

Schwechheimer, S. K., E. Y. Park, J. L. Revuelta, J. Becker, and C. Wittmann. 2016. 'Biotechnology of riboflavin', Appl Microbiol Biotechnol, 100: 2107-19.

Serra, A., P. Strehaiano, and P. Taillandier. 2003. 'Characterization of the metabolic shift of Saccharomyces bayanus var. uvarum by continuous aerobic culture', Appl Microbiol Biotechnol, 62: 564-8.

Sollner, S., S. Deller, P. Macheroux, and B. A. Palfey. 2009. 'Mechanism of flavin reduction and oxidation in the redox-sensing quinone reductase Lot6p from Saccharomyces cerevisiae', Biochemistry, 48: 8636-43.

Stephanopoulos, G. 1999. 'Metabolic fluxes and metabolic engineering', Metab Eng, 1: 1-11.

Suarez-Mendez, C. A., M. Hanemaaijer, A. Ten Pierick, J. C. Wolters, J. J. Heijnen, and S. A. Wahl. 2016. 'Interaction of storage carbohydrates and other cyclic fluxes with central metabolism: A quantitative approach by non-stationary (13)C metabolic flux analysis', Metab Eng Commun, 3: 52-63.

Toya, Y., and H. Shimizu. 2013. 'Flux analysis and metabolomics for systematic metabolic engineering of microorganisms', Biotechnol Adv, 31: 818-26.

van Dam, J. C., C. Ras, and A. ten Pierick. 2011. 'Analysis of glycolytic intermediates with ion chromatography- and gas chromatography-mass spectrometry', Methods Mol Biol, 708: 131-46.

van Eunen, K., S. Rossell, J. Bouwman, H. V. Westerhoff, and B. M. Bakker. 2011. 'Quantitative Analysis of Flux Regulation through Hierarchical Regulation Analysis', Methods in Enzymology, Vol 500, 500: 571-95.

van Winden, W. A., J. C. van Dam, C. Ras, R. J. Kleijn, J. L. Vinke, W. M. van Gulik, and J. J. Heijnen. 2005. 'Metabolic-flux analysis of Saccharomyces cerevisiae CEN.PK113-7D based on mass isotopomer measurements of (13)C-labeled primary metabolites', FEMS Yeast Res, 5: 559-68. 
Weitzel, M., K. Noh, T. Dalman, S. Niedenfuhr, B. Stute, and W. Wiechert. 2013.

'13CFLUX2--high-performance software suite for (13)C-metabolic flux analysis', Bioinformatics, 29: 143-5.

Wiechert, W. 2001. '13C metabolic flux analysis', Metab Eng, 3: 195-206.

Willke, T., and K. D. Vorlop. 2004. 'Industrial bioconversion of renewable resources as an alternative to conventional chemistry', Applied Microbiology and Biotechnology, 66: 131-42.

Wu, L., M. R. Mashego, J. C. van Dam, A. M. Proell, J. L. Vinke, C. Ras, W. A. van Winden, W. M. van Gulik, and J. J. Heijnen. 2005. 'Quantitative analysis of the microbial metabolome by isotope dilution mass spectrometry using uniformly 13C-labeled cell extracts as internal standards', Anal Biochem, 336: 164-71.

Yoo, H., M. R. Antoniewicz, G. Stephanopoulos, and J. K. Kelleher. 2008. 'Quantifying reductive carboxylation flux of glutamine to lipid in a brown adipocyte cell line', J Biol Chem, 283: 20621-7.

Young, J. D. 2014. 'INCA: a computational platform for isotopically non-stationary metabolic flux analysis', Bioinformatics, 30: 1333-5.

Zhang, Y. P., J. Sun, and Y. Ma. 2017. 'Biomanufacturing: history and perspective', J Ind Microbiol Biotechnol, 44: 773-84.

Zheng, Y., Y. Chang, S. Xie, J. Song, and M. Wang. 2018. 'Impacts of bioprocess engineering on product formation by Acetobacter pasteurianus', Appl Microbiol Biotechnol, 102: 2535-41. 


\section{Supplementary Files}

This is a list of supplementary files associated with this preprint. Click to download.

- Supplementarymaterial1.docx

- Supplementarymaterial2.docx 